\title{
AVALIAÇÃO DA QUALIDADE DA ÁGUA DE NASCENTES NA BACIA HIDROGRÁFICA DO ARROIO ANDRÉAS, RS, UTILIZANDO VARIÁVEIS FÍSICAS, QUÍMICAS E MICROBIOLÓGICAS
}

\author{
Priscila Fernandes de Oliveira ${ }^{1}$ \\ Dionei Delevati ${ }^{2}$ \\ Adilson Ben da Costa ${ }^{3}$ \\ Eduardo A. Lobo ${ }^{4}$
}

\section{RESUMO}

O objetivo principal deste estudo foi avaliar a eficiência da implantação de áreas de preservação de nascentes da Bacia Hidrográfica do Arroio Andréas, Vera Cruz, RS, através do Pagamento de Serviços Ambientais (PSA), utilizando um programa de monitoramento ambiental, considerando algumas variáveis físicas, químicas e microbiológicas. O PSA vincula-se ao projeto "Protetor das Águas", que visa a garantir a preservação dos recursos hídricos da referida bacia mediante o pagamento aos agricultores de pequenas propriedades pelo fornecimento de serviços ambientais de proteção das nascentes e áreas ripárias que se situam em suas propriedades. Desta forma, ao longo desta bacia foram selecionadas 20 estações de amostragem para coleta mensais de amostras d'água no período compreendido entre agosto de 2012 a julho de 2013, a fim de medir as variáveis ambientais. As amostras foram analisadas em laboratório e classificadas quanto à qualidade da água segundo a portaria 357/2005 do Conselho Nacional do Meio Ambiente (CONAMA). Verificou-se que das 240 amostras coletadas, $41 \%$ (98 amostras) foram enquadradas nas Classes de uso 3 e 4 do CONAMA, que correspondem a pontos críticos por apresentar uma qualidade da água imprópria para usos múltiplos, tais como água para consumo humano após tratamento simplificado, proteção das comunidades aquáticas e recreação de contato primário (balneabilidade), em função das variáveis ambientais que foram medidas. Este diagnóstico, entretanto, corresponde à caracterização ambiental inicial destas fontes d'água nesta bacia, uma vez que a proteção destas áreas de preservação feita pelo projeto "Protetor das Águas", durante o ano de 2012, atingiu um percentual de $73 \%$ do total, sendo que os $27 \%$ restantes de proteção foram concluídos durante o primeiro semestre de 2013. Desta forma, estas áreas de nascentes e matas ripárias estavam sujeitas ao impacto de uma série de atividades antrópicas locais, como por exemplo, o aporte de nutrientes e carga orgânica oriundos de esgoto doméstico e criação de animais, bem como do excesso de fertilizantes agrícolas utilizados em lavouras.

Palavras-chave: Bacia Hidrográfica do Arroio Andreas, RS. Pagamento por Serviços Ambientais (PSA). Preservação de nascentes. Qualidade da água.

\footnotetext{
${ }^{1}$ Acadêmica do Curso de Engenharia Ambiental da Universidade de Santa Cruz do Sul - UNISC. <rifernandes2010@hotmail.com>

${ }^{2}$ Professor do Departamento de Engenharia, Arquitetura e Ciências Agrárias da Universidade de Santa Cruz do Sul - UNISC. <dionei@unisc.br>

${ }^{3}$ Professor do Departamento de Biologia e Farmácia da Universidade de Santa Cruz do Sul - UNISC. <adilson@unisc.br>

${ }^{4}$ Professor do Departamento de Biologia e Farmácia da Universidade de Santa Cruz do Sul - UNISC. <lobo@unisc.br>
} 


\section{ABSTRACT}

The main objective of this study was to evaluate the efficiency of implementation of conservation areas in the headwaters of the Hydrographical Basin of Andreas Stream, Vera Cruz, RS, through the Payment for Environmental Services (PES), using an environmental monitoring program, considering some physical, chemical and microbiological variables. The PES is linked to the project "Protector of the Waters", which aims to ensure the preservation of the water resources of this basin, based on the payment to the farmers for providing environmental protection services of springs and riparian areas that lie in their properties. Thus, 20 sampling stations were selected along this basin for collecting water samples monthly during the period of August 2012 to July 2013, in order to measure the environmental variables. The samples were analyzed in the laboratory and classified into water quality classes, following the decree 357/2005 of the National Environmental Council (CONAMA). It was found that of 240 samples collected, $41 \%$ (98 samples) were classified in classes of water uses 3 and 4 of CONAMA, which correspond to critical points by presenting a quality of water unfit for multiple uses, such water for human drinking purposes after simplified treatment, protection of aquatic communities and primary contact recreation (bathing), according to the environmental variables that were measured. This diagnosis, however, corresponds to the initial environmental characterization of these headwaters areas in this basin, since the protection of these conservation areas made by the project "Protector of the Waters", during the year 2012, reached a percentage of $73 \%$ of the total, with the remaining $27 \%$ of protection completed during the first half of 2013 . Thus, these headwater areas and riparian forests were subject to the impact of a number of local anthropogenic activities, such as the supply of nutrients and organic matter originating from domestic sewage and livestock, as well as excess of fertilizers used in agricultural crops.

Keywords: Hydrographical Basin of Andreas Stream, RS. Payment for Environmental Services (PES). Headwaters preservation. Water quality.

\section{INTRODUÇÃO}

No Brasil, a água é considerada um recurso natural abundante quando comparada à atual demanda, embora existam áreas muito secas onde a água é tão preciosa que é utilizada apenas para atender às necessidades humanas. Apesar de ser o detentor de $8 \%$ de toda a água doce do mundo, a situação no país é delicada. A maioria do volume disponível (80\%) está na região amazônica e o remanescente (20\%) encontra-se desigualmente dividido entre as demais regiões, nas quais vivem $95 \%$ da população brasileira. Na região Sul há escassez de água apesar dos rios de grande porte e vazão. Essa escassez se deve aos efeitos cumulativos dos processos ambientais e usos múltiplos que são de amplo espectro: agricultura, indústrias, piscicultura, navegação e recreação (TUNDISI, 2000).

Cientes desta problemática ambiental, a Universidade de Santa Cruz do Sul (UNISC) em parceria com a empresa Universal Leaf Tabacos e a Fundación Altadis (entidade sem fins lucrativos pertencente ao Grupo Imperial Tobacco) assinaram, em 2011, um contrato para a execução do projeto "Pagamento por Serviços Ambientais (PSA) na Bacia Hidrográfica do 
Arroio Andréas, RS, Brasil", denominado "Projeto Protetor das Águas", a ser desenvolvido pela UNISC num período de cinco anos (2011-2015).

O "Protetor das Águas" visa a proteger as nascentes e áreas ripárias da referida subbacia, visando a garantir a preservação dos recursos hídricos mediante o pagamento aos agricultores de pequenas propriedades pelo fornecimento de serviços ambientais de proteção das nascentes e áreas ripárias que se situam em suas propriedades.

Neste contexto, a presente pesquisa objetivou avaliar a eficiência da implantação destas áreas de preservação na Bacia Hidrográfica do Arroio Andréas, RS, no período de agosto de 2012 a julho de 2013, utilizando programas de monitoramento ambiental (físico, químico, e microbiológico).

\section{MATERIAL E MÉTODOS}

\section{1 Área de estudo/amostragem}

Localizada na região central do Estado do Rio Grande do Sul, a Bacia Hidrográfica do Arroio Andréas está inserida no município de Vera Cruz, como pode ser visto na Figura 1. Apresenta uma área de drenagem de $80,2 \mathrm{~km}^{2}$, contando com uma população urbana de 11.183 habitantes e uma população rural de 2.964 habitantes (IBGE, 2011). O uso do solo é caracterizado pela agricultura, inclusive com a presença de arroz irrigado.

Ao longo desta Bacia Hidrográfica foram selecionados 20 pontos de coleta para a realização de estudos de monitoramento ambiental (Figura 1), localizados em propriedades rurais que aderiram ao projeto "Protetor das Águas", protegendo estas áreas e recebendo pagamento pelo fornecimento deste Serviço Ambiental (PSA). A localização destes pontos de coleta apresenta-se na Tabela 1.

Tabela 1 - Identificação dos 20 pontos de coleta do Projeto “Protetor das Águas”.

\begin{tabular}{ccc} 
Ponto & \multicolumn{1}{c}{ Coordenada UTM } \\
\cline { 2 - 3 } & 1 & $348010 \mathrm{E}, 6724840 \mathrm{~S}$ \\
2 & $348629 \mathrm{E}, 6723933 \mathrm{~S}$ \\
3 & $348643 \mathrm{E}, 6723474 \mathrm{~S}$ \\
4 & $348478 \mathrm{E}, 6724492 \mathrm{~S}$ \\
5 & $350020 \mathrm{E}, 6722988 \mathrm{~S}$ \\
6 & $349376 \mathrm{E}, 6721851 \mathrm{~S}$ \\
7 & $349750 \mathrm{E}, 6721136 \mathrm{~S}$ \\
8 & $351486 \mathrm{E}, 6722020 \mathrm{~S}$ \\
9 & $356068 \mathrm{E}, 6717901 \mathrm{~S}$ \\
10 & $355566 \mathrm{E}, 6716687 \mathrm{~S}$ \\
11 & $356065 \mathrm{E}, 6716410 \mathrm{~S}$ \\
12 & $355342 \mathrm{E}, 6715608 \mathrm{~S}$ \\
\hline
\end{tabular}




\begin{tabular}{ll}
\hline 13 & $349052 \mathrm{E}, 6722977 \mathrm{~S}$ \\
14 & $352753 \mathrm{E}, 6718520 \mathrm{~S}$ \\
15 & $351218 \mathrm{E}, 6718128 \mathrm{~S}$ \\
16 & $349711 \mathrm{E}, 6718326 \mathrm{~S}$ \\
17 & $350251 \mathrm{E}, 6723933 \mathrm{~S}$ \\
18 & $350227 \mathrm{E}, 6717475 \mathrm{~S}$ \\
19 & $349886 \mathrm{E}, 6717483 \mathrm{~S}$ \\
20 & $353975 \mathrm{E}, 6715567 \mathrm{~S}$ \\
\hline
\end{tabular}

Figura 1 - Mapa da área de estudo mostrando a localização da Bacia Hidrográfica do Arroio Andréas, em relação ao Estado do Rio Grande do Sul, destacando os pontos de coleta selecionados.

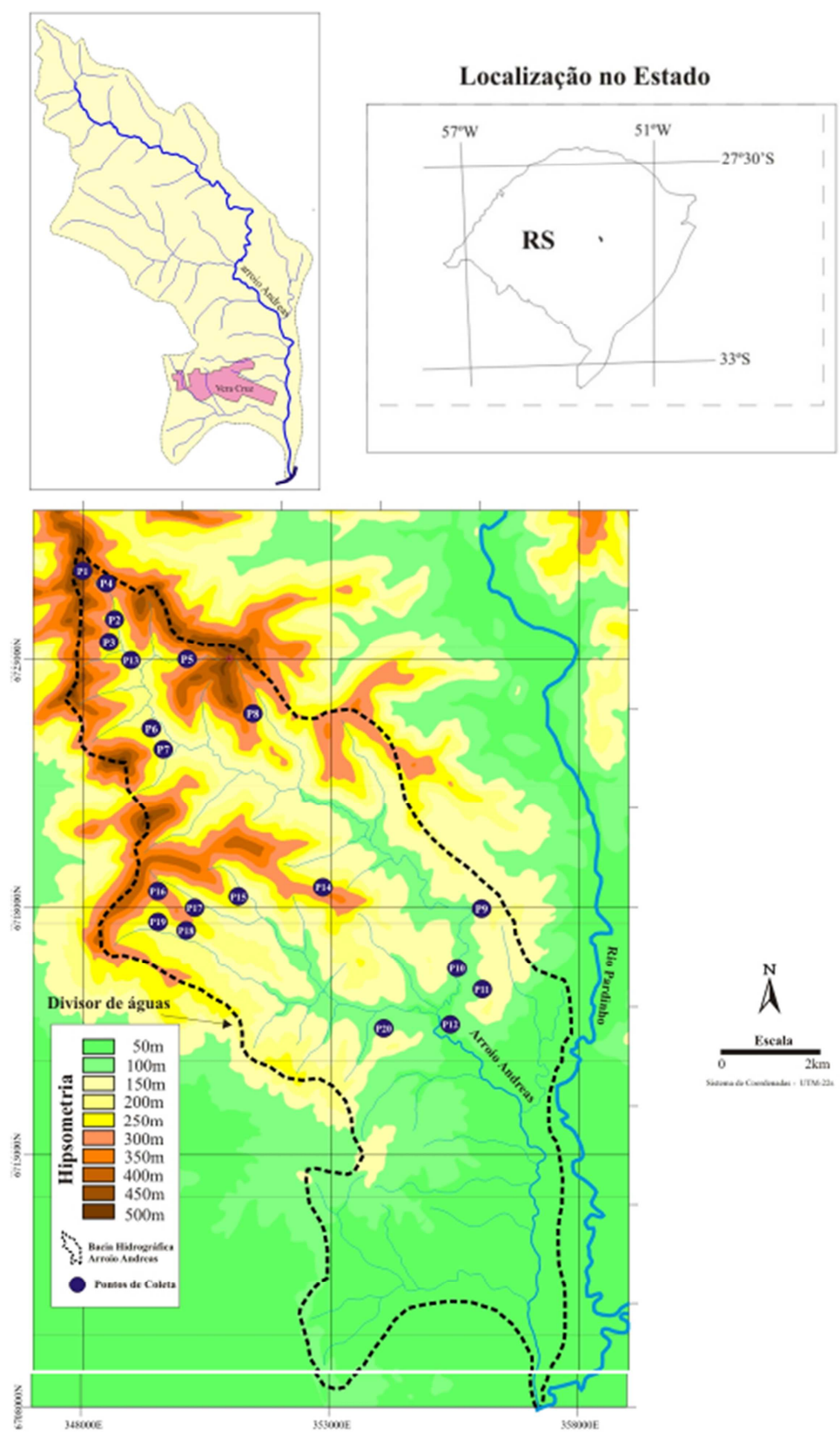


Nos 20 pontos de coleta selecionados foram realizadas coletas mensais para análises de variáveis físicas, químicas e microbiológicas no período compreendido entre agosto de 2012 e julho de 2013. Os seguintes parâmetros de avaliação ambiental foram medidos: temperatura, $\mathrm{pH}$, turbidez, oxigênio dissolvido, demanda bioquímica de oxigênio após cinco dias, nitrato, nitrito, nitrogênio amoniacal total, fosfato, sólidos totais dissolvidos e coliformes termotolerantes. As técnicas utilizadas na coleta das amostras e na determinação analíticas das variáveis físicas, químicas e microbiológicas encontram-se descritas em APHA (2005).

Com base nas análises ambientais, a avaliação da qualidade da água foi feita utilizando a resolução n ${ }^{0} 357$ do Conselho Nacional do Meio Ambiente (CONAMA), de 2005 (BRASIL, 2005), que classifica as águas doces em cinco classes diferentes em função dos usos a que se destinam. Estes dados estão apresentados na Tabela 2.

Tabela 2 - Classificação de águas doces em função dos usos (BRASIL, 2005).

\begin{tabular}{ll}
\hline Classe & Águas que podem ser destinadas \\
\hline Especial & ao abastecimento para consumo humano, com desinfecção; \\
& à preservação do equilíbrio natural das comunidades aquáticas; e, \\
& à preservação dos ambientes aquáticos em unidades de conservação de proteção integral. \\
\hline Classe 1 & ao abastecimento para consumo humano, após tratamento simplificado; \\
& à proteção das comunidades aquáticas; \\
& à recreação de contato primário, tais como natação, esqui aquático e mergulho, conforme \\
& Resolução CONAMA no 274, de 2000; \\
& à irrigação de hortaliças que são consumidas cruas e de frutas que se desenvolvam rentes \\
& ao solo e que sejam ingeridas cruas sem remoção de película; e \\
& à proteção das comunidades aquáticas em Terras Indígenas. \\
\hline Classe 2 & ao abastecimento para consumo humano, após tratamento convencional; \\
& à proteção das comunidades aquáticas; \\
& à recreação de contato primário, tais como natação, esqui aquático e mergulho, conforme \\
& Resolução CONAMA no 274, de 2000; \\
& à irrigação de hortaliças, plantas frutíferas e de parques, jardins, campos de esporte e \\
& lazer, com os quais o público possa vir a ter contato direto; e \\
à aquicultura e à atividade de pesca.
\end{tabular}

\section{RESULTADOS E DISCUSSÃO}

Os resultados da avaliação da qualidade da água utilizando a Resolução 357/2005 do CONAMA (BRASIL, 2005), nos 20 pontos de coleta distribuídos ao longo da Bacia Hidrográfica do Arroio Andréas, RS, no período compreendido entre agosto de 2012 e julho de 2013, apresentam-se na Tabela 3 e Tabela 4. 
Tabela 3 - Classes de uso da água segundo a Resolução CONAMA 357/2005 (BRASIL, 2005), nos pontos de coleta 1 a 10 distribuídos ao longo da Bacia Hidrográfica do Arroio Andréas, RS, no período compreendido entre agosto de 2012 a julho de 2013.

\begin{tabular}{|c|c|c|c|c|c|c|c|c|c|c|}
\hline Datas de coleta & P1 & $\mathbf{P 2}$ & $\mathbf{P 3}$ & $\mathbf{P 4}$ & P5 & $\mathbf{P 6}$ & P7 & P8 & P9 & P10 \\
\hline ago/12 & 4 & 2 & 2 & 2 & 2 & 3 & 2 & 4 & 2 & 1 \\
\hline set/12 & 2 & 1 & 2 & 2 & 1 & 3 & 3 & 3 & 3 & 3 \\
\hline out/12 & 1 & 1 & 4 & 4 & 2 & 4 & 2 & 4 & 1 & 2 \\
\hline nov/12 & 4 & 4 & 3 & 3 & 3 & 4 & 3 & 4 & 2 & 1 \\
\hline $\mathrm{dez} / 12$ & 4 & 2 & 4 & 4 & 2 & 2 & 4 & 4 & 2 & 3 \\
\hline $\mathrm{jan} / 13$ & 3 & 2 & 2 & 4 & 2 & 2 & 1 & 3 & 1 & 1 \\
\hline fev/13 & 4 & 4 & 2 & 3 & 2 & 4 & 1 & 3 & 1 & 3 \\
\hline $\mathrm{mar} / 13$ & 1 & 2 & 3 & 1 & 1 & 3 & 1 & 2 & 1 & 1 \\
\hline $\mathrm{abr} / 13$ & 3 & 3 & 2 & 1 & 1 & 2 & 2 & 4 & 1 & 2 \\
\hline mai/13 & 4 & 2 & 4 & 2 & 2 & 4 & 2 & 2 & 2 & 2 \\
\hline jun $/ 13$ & 1 & 1 & 2 & 1 & 3 & 3 & 2 & 3 & 1 & 2 \\
\hline $\mathrm{jul} / 13$ & 1 & 1 & 2 & 1 & 2 & 3 & 1 & 2 & 1 & 1 \\
\hline $\begin{array}{c}\text { Resolução 357/2005 } \\
\text { (BRASIL, 2005) }\end{array}$ & $\begin{array}{c}\text { Classe } \\
\text { Especial }\end{array}$ & $\begin{array}{c}\text { Classe } \\
1\end{array}$ & $\begin{array}{c}\text { Classe } \\
2\end{array}$ & $\begin{array}{c}\text { Classe } \\
3\end{array}$ & $\begin{array}{c}\text { Classe } \\
4\end{array}$ & & & & & \\
\hline
\end{tabular}

Tabela 4 - Classes de uso da água segundo a Resolução CONAMA 357/2005 (BRASIL, 2005), nos pontos de coleta 11 a 20 distribuídos ao longo da Bacia Hidrográfica do Arroio Andréas, RS, no período compreendido entre agosto de 2012 a julho de 2013.

\begin{tabular}{|c|c|c|c|c|c|c|c|c|c|c|}
\hline Datas de coleta & P11 & P12 & P13 & P14 & P15 & P16 & P17 & P18 & P19 & $\mathbf{P 2 0}$ \\
\hline ago/12 & 2 & 2 & 4 & 3 & 3 & 1 & 2 & 2 & 4 & 2 \\
\hline set/12 & 2 & 3 & 1 & 3 & 3 & 3 & 3 & 3 & 4 & 3 \\
\hline out/12 & 2 & 4 & 2 & 3 & 1 & 3 & 2 & 1 & 1 & 2 \\
\hline nov/12 & 4 & 2 & 2 & 3 & 2 & 2 & 2 & 3 & 1 & 4 \\
\hline $\mathrm{dez} / 12$ & 3 & 4 & 3 & 2 & 4 & 3 & 2 & 4 & 3 & 3 \\
\hline $\mathrm{jan} / 13$ & 3 & 3 & 2 & 3 & 4 & 3 & 1 & 4 & 1 & 2 \\
\hline fev/13 & 2 & 2 & 1 & 2 & 3 & 3 & 1 & 4 & 1 & 4 \\
\hline $\mathrm{mar} / 13$ & 1 & 2 & 1 & 3 & 1 & 2 & 1 & 1 & 1 & 4 \\
\hline $\mathrm{abr} / 13$ & 4 & 2 & 1 & 2 & 1 & 4 & 2 & 4 & 1 & 3 \\
\hline mai/13 & 4 & 4 & 3 & 3 & 2 & 4 & 3 & 4 & 2 & 2 \\
\hline jun/13 & 2 & 2 & 2 & 2 & 2 & 2 & 1 & 2 & 2 & 2 \\
\hline $\mathrm{jul} / 13$ & 1 & 2 & 1 & 3 & 1 & 1 & 1 & 2 & 2 & 2 \\
\hline $\begin{array}{l}\text { Resolução 357/2005 } \\
\text { (BRASIL, 2005) }\end{array}$ & \begin{tabular}{|c|} 
Classe \\
Especial \\
\end{tabular} & $\begin{array}{c}\text { Classe } \\
1 \\
\end{array}$ & $\begin{array}{c}\text { Classe } \\
2 \\
\end{array}$ & $\begin{array}{c}\text { Classe } \\
3 \\
\end{array}$ & $\begin{array}{c}\text { Classe } \\
4 \\
\end{array}$ & & & & & \\
\hline
\end{tabular}

De forma geral, verificou-se que $24 \%$ dos pontos de coleta enquadraram-se nas classes de uso da água do CONAMA como "Classe 1", 35\% como "Classe 2", 23\% como "Classe 3" e $18 \%$ como "Classe 4". 
Os pontos amostrais classificados como pertencendo à Classe de Uso 1 do CONAMA (24\%) caracterizam-se como águas de boa qualidade, apropriadas para o consumo humano após tratamento simplificado, para a proteção das comunidades aquáticas e para a recreação de contato primário (balneabilidade), dentre as suas principais características (Tabela 2). Por sua vez, os pontos amostrais enquadrados como pertencendo à Classe de Uso 2 do CONAMA (35\%) correspondem também a águas de boa qualidade, sendo que a principal diferença com a Classe de Uso 1 do CONAMA é que neste caso a água pode ser usada para fins de consumo humano, entretanto após tratamento convencional.

Já os pontos amostrais enquadrados como pertencendo à Classe de Uso 3 do CONAMA (23\%) apresentam usos bem mais restritivos do que a Classe 2, limitando-se ao consumo humano após tratamento convencional ou avançado, recreação de contato secundário e dessedentação de animais, dentre as suas principais características. Desta forma, usos mais nobres como consumo humano após tratamento simplificado, proteção das comunidades aquáticas e recreação de contato primário (balneabilidade) não são permitidos. As variáveis demanda bioquímica de oxigênio, oxigênio dissolvido, fósforo, turbidez, e coliformes termotolerantes foram as responsáveis por esta classificação, uma vez que os valores máximos permitidos pela resolução para esta classe foram ultrapassados (BRASIL, 2005).

Por último, os pontos amostrais enquadrados como pertencendo à Classe de Uso 4 do CONAMA (18\%) correspondem à classe de pior qualidade, destinada apenas à navegação e harmonia paisagística. As variáveis Demanda Bioquímica de Oxigênio, Oxigênio Dissolvido, Fósforo, Turbidez, Nitrato e Coliformes Termotolerantes foram as responsáveis por esta classificação, uma vez que os valores máximos permitidos pela resolução para esta classe foram ultrapassados (BRASIL, 2005).

De forma geral, verificou-se que das 240 amostras coletadas, $41 \%$ foram enquadradas nas Classes de uso 3 e 4, que correspondem a pontos críticos por apresentar uma qualidade da água fora dos padrões desejados, isto é, classes de uso 1 e 2 (59\% do total de amostras coletadas). Este diagnóstico corresponde à caracterização ambiental inicial das fontes d'água na Bacia Hidrográfica do Arroio Andréas, uma vez que o cercamento destas áreas de preservação por parte do projeto "Protetor das Águas" durante o ano de 2012 atingiu um percentual de $73 \%$ do total, sendo que $27 \%$ do cercamento restante foi concluído durante o primeiro semestre de 2013. Desta forma, estas áreas de nascentes e matas ripárias estavam sujeitas ao impacto de uma série de atividades antrópicas locais, como por exemplo o aporte 
de nutrientes e carga orgânica oriundos de esgoto doméstico e criação de animais, bem como do excesso de fertilizantes e insumos agrícolas utilizados em lavouras.

De fato, estes impactos ambientais podem ser verificados a partir das variáveis que foram responsáveis pela classificação de $41 \%$ das amostras nas classes de uso 3 e 4 , sendo elas a demanda bioquímica de oxigênio, oxigênio dissolvido, fósforo, turbidez, nitrato e coliformes termotolerantes. Assim, em função destas variáveis, conclui-se que a contaminação orgânica e a eutrofização da água são os principais problemas ambientais que caracterizaram estes corpos da água.

Estes resultados vêm corroborar trabalhos de monitoramento ambiental em sistemas hídricos regionais realizados pela UNISC, os quais têm demonstrado que estes já apresentam estados bastante avançados de poluição orgânica e eutrofização (BES et al., 2012; BOHM et al., 2013; DÜPONT et al., 2007; HERMANY et al., 2006; LOBO, 2013; LOBO et al., 2004a,b,c,d; LOBO et at., 2010; OLIVEIRA et al., 2001; SALOMONI et al., 2006; 2011; SCHUCH et al., 2012; WETZEL et al., 2002). Ainda, segundo Tundisi (2006), esta condição caracteriza de forma generalizada os cursos d'água em toda a região Sul do Brasil, conforme resultados obtidos pelo Projeto Brasil das Águas.

\section{CONCLUSÕES}

Conclui-se, desta forma, que a avaliação da qualidade da água das nascentes e áreas ripárias que já estão protegidas, durante o ano de 2014, se constitui no fator chave para chancelar a importância da preservação destes recursos hídricos, assumindo como hipótese de trabalho que, em função desta ação de preservação, a qualidade da água deverá melhorar significativamente, particularmente das 98 amostras (41\%) que foram classificadas nas classes de uso 3 e 4 do CONAMA.

\section{AGRADECIMENTOS}

Ao $\mathrm{CNPq}$ pelo apoio financeiro do projeto "Monitoramento ambiental de áreas de preservação de nascentes da Bacia Hidrográfica do Arroio Andréas, RS, Brasil, implantadas através de Pagamento por Serviços Ambientais (PSA's)", Edital MCT/CNPq/Universal - n $^{\circ}$ 14/2011. Ainda, ao CNPq pela concessão de Bolsa de Iniciação Científica PIBITI/CNPq à primeira autora e ao FAP - Fundo de Apoio da Pesquisa da Universidade de Santa Cruz do Sul. 


\section{REFERÊNCIAS}

APHA. Standard Methods for the Examination of water and Wastewater. 21 ed. Washington: American Public Health Association, 2005

BES, D.; ECTOR, L.; TORGAN, L. C.; LOBO, E. A. Composition of the epilithic diatom flora from a subtropical river, Southern Brazil. Iheringia Série Botânica, v. 67, n. 1, p. 93$125,2012$.

BOHM, J. S.; SCHUCH, M.; DUPONT, A.; LOBO, E. A. Response of Epilithic Diatom Communities to Downstream Nutrient Increases in Castelhano Stream, Venâncio Aires City, RS, Brazil. Journal of Environmental Protection, v. 4, p. 20-26, 2013.

BRASIL. MINISTERIO DO MEIO AMBIENTE. Resolução Conselho Nacional do Meio Ambiente $n^{o}$ 357, de 17 de março de 2005. Ministério do Desenvolvimento Urbano e Meio Ambiente. Diário Oficial da União, Brasília, DF, 18 de março de 2005.

DÜPONT, A.; LOBO, E. A.; COSTA, A. B.; SCHUCH. M. Avaliação da Qualidade da Água do Arroio do Couto, Santa Cruz do Sul, RS, Brasil. Caderno de Pesquisa Série Biologia, Santa Cruz do Sul, v. 19, n. 1, p. 56-74, 2007.

IBGE. Instituto Brasileiro de Geografia e Estatística. Censo Agropecuário. 2011. Disponível em: <http://www.ibge.gov.br/home/estatistica/economia/ppm/2011>. Acesso em: 20 de dez. de 2013.

HERMANY, G.; SCHWARZBOLD, A.; LOBO, E. A.; OLIVEIRA, M. A. Ecology of the epilithic diatom community in a low-order stream system of the Guaíba hydrographical region: subsidies to the environmental monitoring of southern Brazilian aquatic systems. Acta Limnologica Brasiliensia, v. 18, n. 1, p. 25-40, 2006.

LOBO, E. A. O Perifíton como Indicador da Qualidade da Água. p. 205-235. In: SCHWARZBOLD, A., BURLIGA, A, L., TORGAN, L. C. (Orgs.). Ecologia do Perifíton. Rima Editora: São Carlos, SP. 2013.

LOBO, E.; CALLEGARO, V. L. M.; HERMANY, G.; BES, D.; WETZEL, C. E.; OLIVEIRA, M. A. Use of epilithic diatoms as bioindicators, with special emphasis to the eutrophication problem of lotic systems in Southern Brazil. Acta Limnologica Brasiliensia. v. 16 , n. 1, p. 25-40, 2004a.

LOBO, E. A.; CALLEGARO, V. L.; HERMANY, G,; GOMEZ, N.; ECTOR, L. Review of the use of microalgae in South America for monitoring rivers, with special reference to diatoms. Vie et Milieu, France, v. 53, n. 2/3, p. 35-45, 2004 b.

LOBO, E. A.; CALlEGARO, V. L.; WETZEL, C. E.; HERMANY, G. \& BES, D. Water quality study of Condor and Capivara streams, Porto Alegre municipal district, RS, Brazil, using epilithic diatoms biocenoses as bioindicatos. Oceanological and Hydrobiological Studies, Poland, v. 33, n. 2, p. 77-93, 2004c. 
LOBO, E.; BES, D.; TUDESQUE, L.; ECTOR, L. Water quality assessment of the Pardinho River, RS, Brasil, using epilithic diatom assemblages and faecal coliforms as biological indicators. Vie et Milieu, France, v. 54, n. 2/3, p. 115-125, 2004d.

LOBO, E. A.; WETZEL, C. E.; ECTOR, L.; KATOH. K.; BLANCO, S.; MAYAMA, S. Response of epilithic diatom community to environmental gradients in subtropical temperate Brazilian rivers. Limnetica, España, v. 29, n. 2, p. 323-340, 2010.

OLIVEIRA, M. A.; TORGAN, L.; LOBO, E. A.; SCHWARZBOLD, A. Association of eriphitic diatom species on artificial substrate in lotic environments in the Arroio Sampaio basin, RS, Brazil: relationships with abiotic variables. Revista Brasiliera de Biologia, v. 61, n. 4, p. 523540, 2001.

SALOMONI, S.E.; ROCHA, O.; CALLEGARO, V. L. M.; LOBO, E. A. Epilithic diatoms as indicators of water quality in the Gravataí river, Rio Grande do Sul, Brazil. Hydrobiologia, v. 559, p. 233-246, 2006.

SALOMONI, S. E.; ROCHA, O.; HERMANY, G.; LOBO, E. A. Application of water quality biological indices using diatoms as bioindicators in Gravataí River, RS, Brazil. Brazilian Journal of Biology, v. 71, n. 4, p. 949-959, 2011.

SCHUCH, M.; ABREU-JÚNIOR, E.; LOBO, E. A. Water quality of urban streams, Santa Cruz do Sul, Rio Grande do Sul, based on physical, chemical and biological analyses. Bioikos, Campinas, v. 26, n. 1, p. 3-12, 2012.

TUNDISI, J. G. Limnologia e gerenciamento de recursos hídricos: Avanços conceituais e metodológicos. Ciência e Ambiente, Santa Maria, v. 21, p. 9-20, 2000.

TUNDISI, J. G. O Futuro dos Recursos Hídricos no Brasil. Projeto Brasil das Águas. 2006. Disponível em: <http://www.brasildasaguas.com.br/>. Acesso em: 20 de dez. de 2013.

WETZEL, C. E.; LOBO, E. A.; OLIVEIRA, M. A.; BES, D.; HERMANY, G. Diatomáceas epilíticas relacionadas a fatores ambientais em diferentes trechos dos rios Pardo e Pardinho, Bacia Hidrográfica do Rio Pardo, RS, Brasil: Resultados preliminares. Caderno de Pesquisa Série Biologia, Santa Cruz do Sul, v. 14, n. 2, p. 17-38, 2002. 